\title{
Andreev current enhancement and subgap conductance of superconducting $S F N$ hybrid structures in the presence of a small spin-splitting magnetic field
}

\author{
A. Ozaeta, ${ }^{1}$ A. S. Vasenko, ${ }^{2}$ F. W. J. Hekking, ${ }^{3}$ and F. S. Bergeret ${ }^{1,4}$ \\ ${ }^{1}$ Centro de Física de Materiales (CFM-MPC), Centro Mixto CSIC-UPV/EHU, Manuel de Lardizabal 4, E-20018 San Sebastián, Spain \\ ${ }^{2}$ Institut Laue-Langevin, 6 rue Jules Horowitz, BP 156, 38042 Grenoble, France \\ ${ }^{3}$ LPMMC, Université Joseph Fourier and CNRS, 25 Avenue des Martyrs, BP 166, 38042 Grenoble, France \\ ${ }^{4}$ Donostia International Physics Center (DIPC), Manuel de Lardizabal 5, E-20018 San Sebastián, Spain
}

(Received 16 March 2012; revised manuscript received 25 May 2012; published 20 August 2012)

\begin{abstract}
We investigate the subgap transport properties of a $S-F-N_{\mathrm{e}}$ structure. Here $S\left(N_{\mathrm{e}}\right)$ is a superconducting (normal) electrode, and $F$ is either a ferromagnet or a normal wire in the presence of an exchange or a spin-splitting Zeeman field, respectively. By solving the quasiclassical equations we first analyze the behavior of the subgap current, known as the Andreev current, as a function of the field strength for different values of the voltage, temperature, and length of the junction. We show that there is a critical value of the bias voltage $V^{*}$ above which the Andreev current is enhanced by the spin-splitting field. This unexpected behavior can be explained as the competition between two-particle tunneling processes and decoherence mechanisms originating from the temperature, voltage, and exchange field, respectively. We also show that at finite temperature the Andreev current has a peak for values of the exchange field close to the superconducting gap. Finally, we compute the differential conductance and show that its measurement can be used as an accurate way of determining the strength of spin-splitting fields smaller than the superconducting gap.
\end{abstract}

DOI: 10.1103/PhysRevB.86.060509

PACS number(s): 74.45.+c, 74.78.-w, 74.25.F-

Introduction. Transport properties of hybrid structures consisting of superconducting and nonsuperconducting materials have been studied extensively in the past decades. ${ }^{1}$ In particular, there is a renewed interest in the study of the subgap conductance of superconducting hybrid structures in the presence of Zeeman-like fields in view of the presumable detection of Majorana fermions. ${ }^{2}$ Intuitively, due to the gap $\Delta$ in the density of states of a superconductor the charge transport through a superconductor-normal $(S-N)$ metal junction is expected to vanish for voltages smaller than $\Delta$. However, this is not always the case. Experiments on $S / N$ structures have shown a finite subgap conductance. ${ }^{3}$ This behavior was discussed theoretically in Refs. 4 and 5. It was shown that the conductance of a $S-N-N_{\mathrm{e}}$ structure, where $N_{\mathrm{e}}$ denotes a normal metal electrode, shows a peak at a voltage smaller than the superconducting gap ${ }^{6}$ in the case of finite $S / N$ barrier resistances or if $N$ is a diffusive metal. ${ }^{4,5}$ A similar behavior was predicted if one substitutes the normal by a ferromagnetic metal $(F) .^{7-9}$ In all these examples, the key mechanism to explain the finite subgap conductance is the Andreev reflection ${ }^{10,11}$ which takes place at the $S / N$ and $S / F$ interfaces and allows the flow of an electric current even for voltages smaller than the superconducting gap $\Delta$. By this process an electron from the normal region is reflected as a hole forming a coherent electron-hole pair which penetrates into a diffusive normal region over distances of the order of the thermal length $\sqrt{\mathcal{D} / T}$, where $\mathcal{D}$ is the diffusion coefficient and $T$ is the temperature (here and below we set $\hbar=k_{B}=1$ ). This mechanism leads to a finite condensate density in the normal metal, i.e., to the so-called superconducting proximity effect.

At a $S / F$ interface the mechanism of charge transport is however modified since the incoming electron and reflected hole belong to different spin bands. ${ }^{12}$ Thus, one expects a suppression of the Andreev current by increasing the exchange field $h$ of the ferromagnet, which is a measure for the spin splitting at the Fermi level. In the ferromagnet the coherence length of the electron-hole pairs is given by the minimum between the thermal and the magnetic $(\sim \sqrt{\mathcal{D} / h})$ lengths. One expects that by increasing the strength of the field $h$ the electron-hole coherence would be suppressed and hence the subgap current reduced. As we show below, this intuitive picture does not hold always.

In this Rapid Communication we analyze the Andreev current and conductance through a $S-F-N_{\mathrm{e}}$ hybrid structure as a function of the field $h$. Here $h$ denotes either the intrinsic exchange field of a ferromagnet or a spin-splitting field in a normal metal caused by either an external magnetic field or the proximity of an insulating ferromagnet. ${ }^{13} \mathrm{We}$ focus our study on weak fields, $h \lesssim \Delta$ and $h \gtrsim \Delta$. We find an interesting interplay between phase-coherent diffusive propagation of Andreev pairs due to the proximity effect and decoherence mechanisms originated from the temperature, voltage, and exchange field, respectively. This interplay leads to a nonmonotonic behavior of the transport properties as a function of $h$. For very low temperatures and voltages $e V \ll \Delta$ the Andreev current decays monotonically by increasing $h$, as expected. If one keeps the voltage low but now increases the temperature, the Andreev current shows a peak at $h \approx \Delta$. An unexpected behavior is obtained when the voltage exceeds some critical value $V^{*}$. In this case, the Andreev current is enhanced by the field $h$ reaching a maximum at $h \approx e V$. We show that the value of $V^{*}$ depends on the length of the $F$ wire and the temperature. In particular, for zero temperature and in the long-junction limit, i.e., when the length of $F$ is much larger than the coherence length, we show that $e V^{*} \approx 0.56 \Delta_{0}$, where $\Delta_{0}$ is the value of $\Delta$ at $T=0$. We also compute the subgap conductance of the system at low temperatures and small fields $h<\Delta$. We show that it has a peak at $e V=h$. Thus, transport measurements of this type can be used to 
determine the strength of a weak exchange or Zeeman-like field in the nanostructure.

Model and basic equations. We consider a ferromagnetic wire $F$. Its length $L$ is smaller than the inelastic relaxation length. The wire is attached at $x=0$ to a superconducting $(S)$ and at $x=L$ to a normal $\left(N_{\mathrm{e}}\right)$ electrode. As noticed above, $F$ can also describe a normal wire in a spin-splitting field $B$ (in which case $h=\mu_{B} B$, where $\mu_{B}$ is the Bohr magneton) or in proximity with an insulating ferromagnet. ${ }^{13}$ We consider the diffusive limit, i.e., we assume that the elastic scattering length is much smaller than the decay length of the superconducting condensate into the $F$ region. In order to describe the transport properties of the system we compute the quasiclassical Green's functions. ${ }^{14,15}$ They obey the Usadel equation ${ }^{16}$ that in the so-called $\theta$ parametrization reads ${ }^{15}$

$$
\partial_{x x}^{2} \theta_{ \pm}=2 i \frac{E \pm h}{\mathcal{D}} \sinh \theta_{ \pm} \cdot
$$

Here the upper (lower) index denotes the spin-up (spin-down) component. The normal and anomalous Green's functions are given by $g_{ \pm}=\cosh \theta_{ \pm}$and $f_{ \pm}=\sinh \theta_{ \pm}$, respectively. Because of the high transparency of the $F / N_{\mathrm{e}}$ interface the functions $\theta_{ \pm}$vanish at $x=L$, i.e., superconducting correlations are negligible at the $F / N_{\mathrm{e}}$ interface. We consider a tunneling barrier at the $S / F$ interface and assume that its tunneling resistance $R_{T}$ is much larger than the normal resistance $R_{F}$ of the $F$ layer. Thus, by voltage biasing the $N_{\mathrm{e}}$ the voltage drop takes place at the $S / F$ tunnel interface. To leading order in $R_{F} / R_{T} \ll 1$ the Green's functions obey the Kupriyanov-Lukichev boundary condition at $x=0,{ }^{17}$

$$
\left.\partial_{x} \theta_{ \pm}\right|_{x=0}=\frac{R_{F}}{L R_{T}} \sinh \left[\left.\theta_{ \pm}\right|_{x=0}-\theta_{S}\right],
$$

where $\theta_{S}=\operatorname{arctanh}(\Delta / E)$ is the superconducting bulk value of the function $\theta$. Once the functions $\theta_{ \pm}$are obtained, one can compute the current through the junction. In particular, we are interested in the Andreev current, i.e., the current for voltages smaller than the superconducting gap due to Andreev processes at the $S / F$ interface. Such current is given by the expression $^{5,18}$

$$
I_{A}=\sum_{j= \pm} \int_{0}^{\Delta} \frac{n_{-}(E) d E / 2 e R_{T}}{2 W \alpha_{j}(E)-\sqrt{1-(E / \Delta)^{2}} \operatorname{Im}^{-1}\left(\left.\sinh \theta_{j}\right|_{x=0}\right)},
$$

where $\quad n_{-}(E)=\frac{1}{2}(\tanh [(E+e V) / 2 T]-\tanh [(E-e V) /$ $2 T])$ is the quasiparticle distribution function in the $N_{\mathrm{e}} \quad$ electrode, $\quad \alpha_{ \pm}(E)=(1 / \xi) \int_{0}^{L} d x \cosh ^{-2}\left[\operatorname{Re} \theta_{ \pm}(x)\right]$, $W=\xi R_{F} / 2 L R_{T}$ is the diffusive tunneling parameter, ${ }^{17,19}$ and $\xi=\sqrt{\mathcal{D} / 2 \Delta}$ is the superconducting coherence length. Equation (3) is the expression used throughout this Rapid Communication in order to determine the subgap charge transport. $^{20}$

Results. We first compute the Andreev current numerically by solving Eqs. (1)-(3). In Fig. 1 we show the dependence of the Andreev current on the exchange field $h$ for different values of the bias voltage and temperature for a ferromagnetic $F$ wire of the length $L=10 \xi$.

We consider first the zero-temperature limit. For small enough voltages [e.g., $e V=0.3 \Delta$, the black solid line in

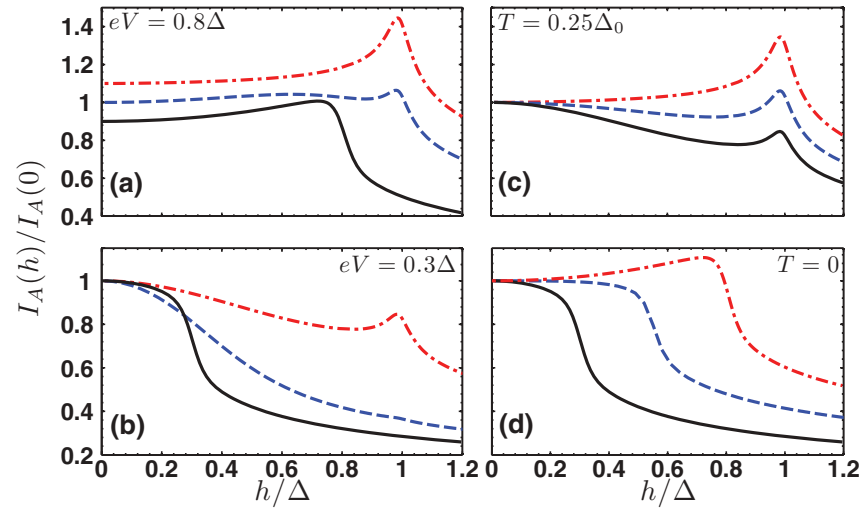

FIG. 1. (Color online) The $h$ dependence of the ratio $I_{A}(h) / I_{A}(0)$ for $L=10 \xi$ and $W=0.007$. The left panels correspond to (a) $\mathrm{eV}=$ $0.8 \Delta$ and (b) $e V=0.3 \Delta$. The different curves are for $T=0$ (black solid line), $T=0.12 \Delta_{0}$ (blue dashed line), and $T=0.25 \Delta_{0}$ (red point-dashed line). The right panels correspond to (c) $T=0.25 \Delta_{0}$ and (d) $T=0$, while the different curves to $e V=0.3 \Delta$ (black solid line), $e V=0.55 \Delta$ (blue dashed line), and $e V=0.8 \Delta$ (red pointdashed line). In (a) the curves are vertically shifted with respect to each other for clarity.

Fig. 1(b)] the Andreev current decays monotonously with increasing $h$. This behavior is the one expected, since by increasing $h$ the coherence length of the Andreev pairs in the normal region is suppressed, leading to a reduction of the subgap current. For large enough voltages (e.g., $e V=0.8 \Delta$ in Fig. 1) and keeping the temperature low, the Andreev current first increases by increasing $h$, reaches a maximum at $h \approx e V$, and then drops by a further increase of the exchange field, as it is shown for example by the black solid line in Fig. 1(a). A common feature of all the low-temperature curves in Fig. 1 is the sharp suppression of the Andreev current at $h \approx e V$.

For large enough temperatures $\left[T=0.25 \Delta_{0}\right.$ in Fig. 1(c) $]$ one observes a peak at $h \approx \Delta$ [Fig. 1(c)]. The relative height of this peak increases with temperature and voltage, as one sees in Figs. 1(a) and 1(c), respectively. In the case of large enough values of $V$ and $T$, one is able to observe both the enhancement of the Andreev current by increasing $h$ and the peak at $h \approx \Delta$ [see, for example, the blue dashed line in Fig. 1(a)]. For values of the exchange field larger than $\Delta$, the Andreev current decreases by increasing $h$ in all cases. In principle all the behaviors of the Andreev current can be observed by measuring the full electric current through the junction as the single-particle current is almost independent of $h$.

In order to give a physical interpretation of these results, we first recall the details of the process of two-electron tunneling that gives rise to subgap current ${ }^{4}$ in diffusive systems in the absence of an exchange field. The value of this current is governed by two competing effects. On the one hand, the origin of the subgap current is the tunneling from the normal metal to the superconductor of two electrons with energies $\xi_{k_{1}}$ and $\xi_{k_{2}}$, respectively, and momenta $k_{1}$ and $k_{2}$, that form a Cooper pair. This process is of second order in tunneling and therefore involves a virtual state with an excitation on both sides of the tunnel barrier. The relevant virtual state energies are given by the difference $E_{k}-\xi_{k_{1}, k_{2}}$, where 


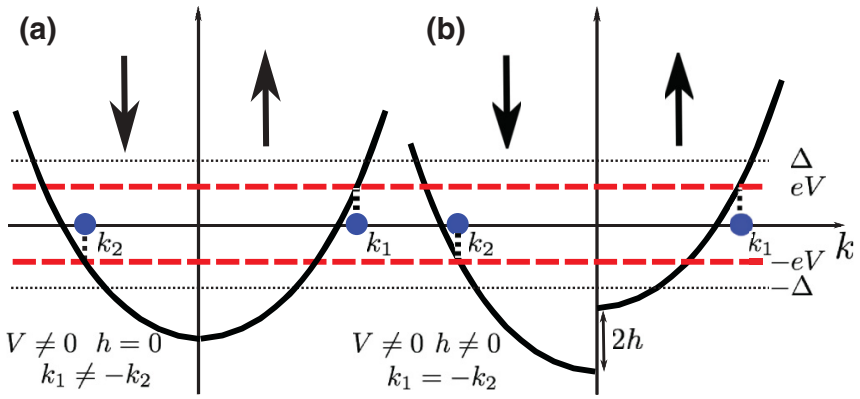

FIG. 2. (Color online) Schematic energy diagram for a nonmagnetic (a) and magnetic (b) metal. The thick solid parabolas are the dispersions of free electrons with spin up $(\uparrow)$ and spin down $(\downarrow)$. The $k$ axis corresponds to the Fermi level in the superconductor. We consider quasielectrons and quasiholes with energies $\pm e V<\Delta$ and momentum $k_{1,2}$. Time-reversal pairing requires that $k_{1}=k_{2}$. In case of a normal metal [(a)] this condition is satisfied only for $e V=0$ while for a ferromagnet $(h \neq 0)$ if $h=e V$.

$E_{k}=\sqrt{\Delta^{2}+\xi_{k}^{2}}$ is the excitation energy of a quasiparticle with the momentum $k$ in the superconductor. Typical values of $\xi_{k}$ are $T$ or $e V$. Hence under subgap conditions $T, e V \ll \Delta$, the virtual state energy is typically given by the superconducting gap $\Delta$. However, when these characteristic energies become larger and approach the value of the gap, the difference $E_{k}-\xi_{k_{1}, k_{2}}$ eventually vanishes. As a result, the amplitude for two-electron tunneling increases drastically, leading to a strong increase of the Andreev current, accompanied by the onset of single-particle tunneling at energies above the gap $\Delta$. On the other hand, two-electron tunneling is a coherent process: the main contribution to two-electron tunneling stems from two nearly time-reversed electrons $k_{1} \simeq-k_{2}$ located in an energy window of width $\delta \epsilon \sim e V, T$ close to the Fermi energy, diffusing phase coherently over a typical distance $L_{\text {coh }}=\sqrt{\mathcal{D} /(\delta \epsilon)}$ in the normal metal before tunneling. ${ }^{4}$ This coherence length decreases upon increasing the characteristic energies $\delta \epsilon \simeq e V, T$, thereby decreasing the Andreev current.

We now turn to the effect of the exchange field $h$ on two-electron tunneling. If $h$ is nonzero, the majority and minority spin electrons at the Fermi level are characterized by different wave vectors $k_{F, \pm}=k_{F} \mp \delta k$, where $\delta k \sim h / v_{F}$ and $v_{F}$ is the Fermi velocity. In Fig. 2 we show a schematic energy diagram. The wave vectors $k_{F, \pm}$ are determined by the intersection between the parabolas and the $k$ axis. For a given value of $e V \lesssim \Delta$ and in the absence of an exchange field the relevant excitations with energies $\sim \pm e V$ and wave vectors $k_{1,2}$ are not time reversed [see Fig. 2(a)] and therefore their contribution to the current is not coherent. However, upon increasing $h,\left|k_{1}\right| \rightarrow\left|k_{2}\right|$, i.e., the relevant excitations become more and more coherent, leading to an additional increase of two-electron tunneling. In particular, when $h=e V, k_{1}=$ $-k_{2}$ [cf. Fig. 2(b)]. If $T \rightarrow 0$ there are no occupied states for $\xi_{k}>e V$. Consequently, as soon as $h>e V$, the energy window around the Fermi level does not contain time-reversed electrons. This leads to the drop of the Andreev current shown, for example, in Fig. 1(d). In contrast, for finite values of $T$, there are thermally induced quasiparticles with energy $\sim \Delta$ that become exactly time reversed whenever $h=\Delta$. This leads to the maximum of the current at $h=\Delta$ when the temperature is finite [cf. Fig. 1(c)]. The effects are most clearly seen when plotting the ratio $I_{A}(h) / I_{A}(0)$, as the Andreev pair decoherence effects due to temperature or voltage are then divided out.

A more quantitative understanding of the effects discussed above can be gotten by analyzing some limiting cases in which simple analytical expressions for the current can be derived. We first focus our analysis on the zero-temperature limit. Due to the tunneling barrier at the $S / F$ interface the proximity effect is weak and hence one can linearize Eqs. (1) and (2) with respect to $R_{F} / R_{T} \ll 1$. After a straightforward calculation one obtains the Andreev current in this limit,

$$
\begin{aligned}
I_{A}= & \frac{W \Delta_{0}^{2}}{2 e R_{T}} \sum_{j= \pm} \int_{0}^{e V} \frac{d E}{\Delta_{0}^{2}-E^{2}} \\
& \times \operatorname{Re}\left[\sqrt{\frac{i \Delta_{0}}{E+j h}} \tanh \left(\sqrt{\frac{E+j h}{i \Delta_{0}}} \frac{L}{\xi}\right)\right] .
\end{aligned}
$$

For a large exchange field, $h \gg \Delta_{0}>e V$ one can evaluate this expression, obtaining

$$
I_{A} \approx \frac{R_{F} \Delta_{0}}{8 e L R_{T}^{2}} \sqrt{\frac{\mathcal{D}}{h}} \log \left[\frac{\Delta_{0}+e V}{\Delta_{0}-e V}\right] .
$$

Thus, the Andreev current decays as $h^{-1 / 2}$ for large values of $h$ in accordance with our numerical results (see Fig. 1).

In the case of small values of $h, h \lesssim e V<\Delta_{0}$, one can evaluate Eq. (4) in the long-junction limit, i.e., when $L \gg$ $\sqrt{D / h}$. In this case the Andreev current reads

$$
I_{A}=\frac{\Delta_{0} \xi R_{F}}{e L R_{T}^{2}} \sum_{j= \pm} \frac{\operatorname{arctanh}\left(\sqrt{\frac{e V+j h}{\Delta_{0}+j h}}\right)+\arctan \left(\sqrt{\frac{e V-j h}{\Delta_{0}+j h}}\right)}{\sqrt{\Delta_{0}+j h}} .
$$

This expression describes the two different behaviors obtained in Fig. 1 for $h \leqslant e V$. For small voltages $I_{A}$ decreases by increasing the field $h$. However, for large enough values of the voltage $I_{A}$ is enhanced by the presence of the field. From Eq. (6) we can determine the voltage $V^{*}$, at which the crossover between these two behaviors takes place, by expanding the expression for the current up to second order in $h / e V \ll 1$, i.e., up to the first nonvanishing correction to $I_{A}$ due to the exchange field. This expansion leads to the following transcendental expression which determines the voltage $V^{*}$ at which the crossover takes place:

$$
\left(\frac{\Delta_{0}}{e V^{*}}\right)^{3 / 2}=\frac{3}{2}\left(\operatorname{arctanh} \sqrt{e V^{*} / \Delta_{0}}+\arctan \sqrt{e V^{*} / \Delta_{0}}\right) .
$$

From here we get $e V^{*} \approx 0.56 \Delta_{0}$. For $V<V^{*}$ the Andreev current decays monotonically with $h$ while for $V>V^{*}$ it increases up to a maximum value at $h \lesssim e V$. This is in agreement with our numerical results in Fig. 1.

For an arbitrary length $L$ and finite temperature we have computed the value of $V^{*}$ numerically. In Fig. 3 we show the results. The solid black line gives the values of $V^{*}$ as a function of $L$ and $T$ [Figs. 3(a) and 3(b), respectively]. The area below the black curve corresponds to the range of parameters for which the Andreev current is suppresses by the presence of a spin-splitting field, while the area above 


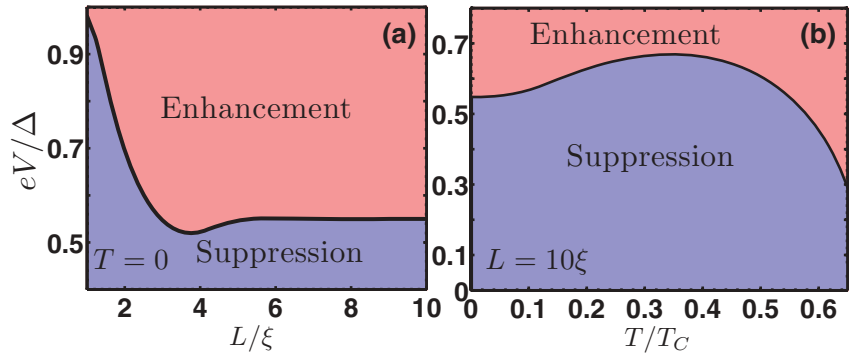

FIG. 3. (Color online) Voltage-junction length (a) and voltagetemperature (b) diagrams. The black solid line represents the values of $e V^{*} / \Delta$. For the range of parameters situated below this line the Andreev current decreases in the presence of a small exchange field (suppression), while in the region above the line the current increases (enhancement). We set $W=0.007$ in both panels, $T=0$ in (a) and $L=10 \xi$ in (b).

the solid line corresponds to the range of parameters for which the unexpected enhancement of the subgap current takes place. According to Fig. 3(a), at $T=0$ the value of $V^{*}$ first decreases as $L$ increases, reaches a minimum, and then grows again up to the asymptotic value $e V^{*} \approx 0.56 \Delta_{0}$. Also the dependence of $V^{*}$ on the temperature is nonmonotonic, having a maximum value at $T \sim 0.2 \Delta_{0}$.

Small spin-splitting fields, as those studied in the present work, can be created by applying an external magnetic field $B$, in which case $h=\mu_{B} B$ or by the proximity of a ferromagnetic insulator as discussed in Ref. 13. It may be also an intrinsic exchange field of weak ferromagnetic alloys (see, for example, Ref. 21). Such small exchange fields are in principle difficult to detect. However, as we show in Fig. 4, by measuring the subgap differential conductance $G=d I / d V$ at low temperatures, one can accurately determine the value of $h$. At $T=0$ the conductance shows two well defined peaks, one at $e V=h$ and the other at $e V=\Delta$. These are related to a sudden increase of the coherence length between the electron-hole pairs in the ferromagnet and of the two-particle tunneling amplitude, respectively. As we have seen above, at small voltages $e V<h$ electrons with majority spins do not find time-reversed partners in the narrow energy window around the Fermi energy, i.e., such pairs show weak coherence. By increasing the voltage $e V<h$, the contribution of timereversed electrons to the current gradually increases and consequently the differential conductance increases, reaching a maximum at $e V=h$. A further increase of the voltage, $e V>h$, leads to an increasing contribution to the current from non-time-reversed electron-hole pairs and therefore to a

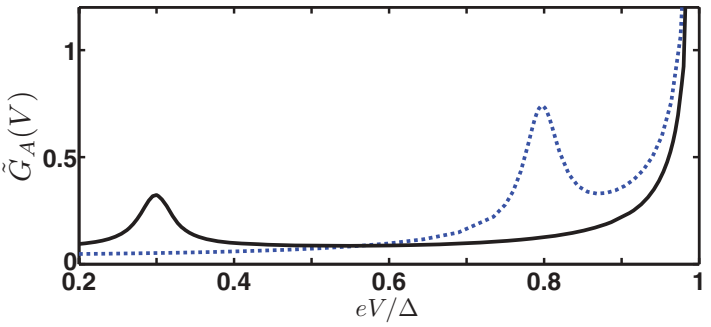

FIG. 4. (Color online) The bias voltage dependence of differential conductance at $T=0$ for fields $h=0.3$ (black solid) and $h=0.8$ (blue dashed). Here $\tilde{G}_{A}=4 R_{T} G_{A}, W=0.007$, and $L=10 \xi$.

suppression of the coherent contribution to $G$. At $h<e V \lesssim \Delta$ the two-electron tunneling amplitude increases as $(e V-\widetilde{\Delta})^{-1}$ due to virtual state contributions with energies $e V$ close to the gap; as a result the conductance shows a sharp increase. For $h \rightarrow 0$ (normal metal) the peak moves toward $e V \rightarrow 0$ (not shown here), which corresponds to the zero bias peak discussed, for example, in Ref. 22.

In conclusion, we present an exhaustive study of the subgap charge current through $S-F-N_{\mathrm{e}}$ hybrid structures in the presence of a spin-splitting field. We have demonstrated the existence of a threshold bias voltage $V^{*}$ above which the Andreev current can be enhanced by an exchange field. We also have shown that at finite temperatures the Andreev current has a peak for values of the exchange field close to the superconducting gap $\Delta$. Finally, we have proposed a way to determine the strength of small exchange fields by measuring the differential conductance. Beyond the fundamental interest, our results can also be useful for the implementation of a recent and interesting proposal ${ }^{13}$ which suggests a way to detect the odd-triplet component ${ }^{23}$ of the superconducting condensate induced in a normal metal in contact with a superconductor and a ferromagnetic insulator. The latter induces an effective exchange field in the normal region. The amplitude of such induced exchange fields is smaller than the superconducting gap. ${ }^{24}$ Therefore the proposed ferromagnet proximity system in Ref. 13 is a candidate to observe the phenomena described in the present work.

Acknowledgments. The authors thank E.I. Kats for useful discussions. F.S.B. and A.O. acknowledge the Spanish Ministry Economy and Competitiveness under Project No. FIS2011-316 28851-C02-02. The work of A.O. was supported by the CSIC and the European Social Fund under the JAEPredoc program.

\footnotetext{
${ }^{1}$ Y. Nazarov and Y. Blanter, Quantum Transport (Cambridge University Press, New York, 2009).

${ }^{2}$ V. Mourik, K. Zuo, S. M. Frolov, S. R. Plissard, E. P. A. M. Bakkers, and L. P. Kouwenhoven, Science 336, 1003 (2012).

${ }^{3}$ A. Kastalsky, A. W. Kleinsasser, L. H. Greene, R. Bhat, F. P. Milliken, and J. P. Harbison, Phys. Rev. Lett. 67, 3026 (1991).

${ }^{4}$ F. W. J. Hekking and Yu. V. Nazarov, Phys. Rev. Lett. 71, 1625 (1993); Phys. Rev. B 49, 6847 (1994).
}

${ }^{5}$ A. F. Volkov, A. V. Zaitsev, and T. M. Klapwijk, Physica C 210, 21 (1993).

${ }^{6}$ Throughout this Rapid Communication $\Delta$ denotes the temperaturedependent BCS gap, while $\Delta_{0}$ is its value at $T=0$.

${ }^{7}$ M. Leadbeater, C. J. Lambert, K. E. Nagaev, R. Raimondi, and A. F. Volkov, Phys. Rev. B 59, 12264 (1999).

${ }^{8}$ R. Seviour, C. J. Lambert, and A. F. Volkov, Phys. Rev. B 59, 6031 (1999). 
${ }^{9}$ T. Yokoyama, Y. Tanaka, and A. A. Golubov, Phys. Rev. B 72, 052512 (2005).

${ }^{10}$ A. F. Andreev, Zh. Eksp. Teor. Fiz. 46, 1823 (1964) [Sov. Phys. JETP 19, 1228 (1964)]; D. Saint-James, J. Phys. (Paris) 25, 899 (1964).

${ }^{11}$ B. Pannetier and H. Courtois, J. Low Temp. Phys. 118, 599 (2000).

${ }^{12}$ M. J. M. de Jong and C. W. J. Beenakker, Phys. Rev. Lett. 74, 1657 (1995)

${ }^{13}$ A. Cottet, Phys. Rev. Lett. 107, 177001 (2011).

${ }^{14}$ A. I. Larkin and Yu. N. Ovchinnikov, in Nonequilibrium Superconductivity, edited by D. N. Langenberg and A. I. Larkin (Elsevier, Amsterdam, 1986).

${ }^{15}$ W. Belzig, F. K. Wilhelm, C. Bruder, G. Schön, and A. D. Zaikin, Superlatt. Microstruct. 25, 1251 (1999).

${ }^{16}$ K. D. Usadel, Phys. Rev. Lett. 25, 507 (1970).

${ }^{17}$ M. Yu. Kuprianov and V. F. Lukichev, Zh. Eksp. Teor. Fiz. 94, 139 (1988) [Sov. Phys. JETP 67, 1163 (1988)].

${ }^{18}$ A. S. Vasenko, E. V. Bezuglyi, H. Courtois, and F. W. J. Hekking, Phys. Rev. B 81, 094513 (2010).

${ }^{19}$ E. V. Bezuglyi, A. S. Vasenko, V. S. Shumeiko, and G. Wendin, Phys. Rev. B 72, 014501 (2005); E. V. Bezuglyi, A. S. Vasenko,
E. N. Bratus, V. S. Shumeiko, and G. Wendin, ibid. 73, 220506(R) (2006).

${ }^{20} \mathrm{We}$ neglect here the contribution to $I_{A}$ from partial Andreev reflection processes at energies above the gap. In the case of tunneling barriers, this contribution leads to small corrections and can be neglected (Ref. 18).

${ }^{21}$ T. Kontos, M. Aprili, J. Lesueur, X. Grison, and L. Dumoulin, Phys. Rev. Lett. 93, 137001 (2004).

${ }^{22}$ B. J. van Wees, P. de Vries, P. Magnée, and T. M. Klapwijk, Phys. Rev. Lett. 69, 510 (1992). In the latter work the effect of a magnetic field on the orbital motion of the electrons was considered. It was shown that for certain critical value of the external field the zero bias peak of the conductance is suppressed. In contrast, we study HERE the effect of a spin-splitting field which is either an intrinsic property of the $\mathrm{F}$ layer or was originated in a normal thin layer or wire in the presence of an external magnetic field. In the latter case orbital effects can be neglected when the applied field is parallel to the plane of the layer.

${ }^{23}$ F. S. Bergeret, A. F. Volkov, and K. B. Efetov, Rev. Mod. Phys. 77, 1321 (2005).

${ }^{24}$ R. Meservey and P. M. Tedrow, Phys. Rep. 238, 173 (1994); X. Hao, J. S. Moodera, and R. Meservey, Phys. Rev. B 42, 8235 (1990). 